\title{
The chiral transitions in heavy-light mesons
}

\author{
A.M.Badalian $*$ Yu.A.Simonov $\dagger$ and M.A.Trusov \\ ITEP, Moscow, Russia
}

(Dated: December 22, 2007)

\begin{abstract}
The mass shifts of the $P$-wave $D_{s}$ and $B_{s}$ mesons due to coupling to $D K, D^{*} K$ and $B K$, $B^{*} K$ channels are studied using the chiral quark-pion Lagrangian without fitting parameters. The strong mass shifts down $\sim 140 \mathrm{MeV}$ and $\sim 100 \mathrm{MeV}$ for $D_{s}^{*}\left(0^{+}\right)$and $D_{s}\left(1^{+^{\prime}}\right)$ and $\sim 100 \mathrm{MeV}$ for $B_{s}^{*}\left(0^{+}\right)$and $B_{s}\left(1^{+^{\prime}}\right)$ are calculated. Two factors are essential for large mass shifts: strong coupling of the $0^{+}$and $1^{+^{\prime}}$ states to the $S$-wave decay channel, containing a Nambu-Goldstone meson, and the chiral flip transitions due to the bispinor structure of both heavy-light mesons. The masses $M\left(B_{s}^{*}\left(0^{+}\right)\right)=5695(10) \mathrm{MeV}$ and $M\left(B_{s}\left(1^{+^{\prime}}\right)\right)=5730(15) \mathrm{MeV}$, very close to $M\left(B\left(0^{+}\right)\right)$ and $M\left(B\left(1^{+^{\prime}}\right)\right)$, are predicted. Experimental limit on the width $\Gamma\left(D_{s 1}(2536)\right)<2.3 \mathrm{MeV}$ puts strong restrictions on admittable mixing angle between the $1^{+}$and $1^{+^{\prime}}$ states, $|\phi|<6^{\circ}$, which corresponds to the mixing angle $\theta$ between the ${ }^{3} P_{1}$ and ${ }^{1} P_{1}$ states, $29^{\circ}<\theta<41^{\circ}$.
\end{abstract}

PACS numbers: 14.40.Lb, 12.39.Fe, 12.40.Yx

*Electronic address: badalian@itep.ru

${ }^{\dagger}$ Electronic address: simonov@itep.ru

‡Electronic address: trusov@itep.ru 


\section{INTRODUCTION}

The heavy-light (HL) mesons play a special role in hadron spectroscopy. First of all, a HL meson is the simplest system, containing one light quark in the field of almost static heavy antiquark, and that allows to study quark (meson) chiral properties. The discovery of the $D_{s}(2317)$ and $D_{s}(2460)$ mesons [1, 2] with surprisingly small widths and low masses has given an important impetus to study chiral dynamics and raised the question why their masses are considerably lower than expected values in different approaches: in relativistic quark model calculations [3]-[6], on the lattice [7], in QCD Sum Rules [8, 9], in chiral models [10]-[12] ( for reviews see also [13, 14]). The masses of $D_{s}\left(0^{+}\right)$and $D_{s}\left(1^{+^{\prime}}\right)$ in closed-channel approximation typically exceed by $\sim 140$ and $90 \mathrm{MeV}$ their experimental numbers.

Thus main theoretical goal is to understand dynamical mechanism responsible for such large mass shifts of the $0^{+}$and $1^{+^{\prime}}$ levels (both states have the light quark orbital angular momentum $l=0$ and $j=1 / 2$ ) and explain why the position of other two levels (with $j=3 / 2$ ) remains practically unchanged. The importance of second fact has been underlined by S.Godfrey in [5].

The mass shifts of the $D_{s}\left(0^{+}, 1^{+^{\prime}}\right)$ mesons have already been considered in a number of papers with the use of unitarized coupled-channel model [15], in nonrelativistic Cornell model [16], and in different chiral models [17]-[19]. Here we address again this problem with the aim to calculate also the mass shifts of the $D_{s}\left(1^{+^{\prime}}\right)$ and $B_{s}\left(0^{+}, 1^{+^{\prime}}\right)$ states and the widths of the $2^{+}$and $1^{+}$states, following the approach developed in [18], for which strong coupling to the S-wave decay channel, containing a pseudoscalar $(P)$ Nambu-Goldstone (NG) meson, is crucially important. Therefore in this approach principal difference exists between vectorvector $(V V)$ and $V P$ (or $P P$ ) channels. This analysis of two-channel system is performed with the use of the chiral quark-pion Lagrangian which has been derived directly from the QCD Lagrangian [20] and does not contain fitting parameters, so that the shift of the $D_{s}^{*}\left(0^{+}\right)$ state $\sim 140 \mathrm{MeV}$ is only determined by the conventional decay constant $f_{K}$.

Here the term "chiral dynamics" implies the mechanism by which in the transition from one HL meson to another the octet of the NG mesons $\phi$ is emitted. The corresponding Lagrangian $\Delta L_{F G M}$,

$$
\Delta L_{F C M}=\bar{q}(\sigma r) \exp \left(i \gamma_{5} \phi / f_{\pi}\right) q
$$

contains the light-quark part $\exp \left(i \gamma_{5} \phi / f_{\pi}\right)$, where $\phi$ is the $S U(3)$ octet of NG mesons and 
the important factor $\gamma_{5}$ is present. In the lowest order in $\phi$ this Lagrangian coincides with well-known effective Lagrangian $\Delta L_{e f f}$ suggested in [21],[22], where, however, an arbitrary constant $g_{A}$ is introduced. At large $N_{c}$, as argued in [21], this constant has to be equal unity, $g_{A}=1$. In [10, 17, 22] this effective Lagrangian was applied to describe decays of HL mesons taking $g_{A}<0.80$.

More general Lagrangian $\Delta L_{F C M}$ (1) was derived in the framework of the field correlator method (FCM) [20, 23], in which the constant $g_{A}=1$ in all cases, and which contains NG mesons to all orders, as seen from its explicit expression (1).

In Appendix A with the use of the Dirac equation we show that in the lowest order in $\phi \Delta L_{F C M}=\Delta L_{e f f}$, if indeed $g_{A}=1$. In our calculations we always use $\Delta L_{F C M}$ with the $g_{A}=1$ and derive the nonlinear equation for the energy shift and width, $\Delta E=\Delta \bar{E}-\frac{\Gamma}{2}$, as in [18]. We do not assume any chiral dynamics for the unperturbed levels, which are calculated here with the use of the QCD string Hamiltonian [24, 25], because the mass shift $\Delta E$ appears to be weakly dependent on the position of unperturbed level.

It is essential that resulting shifts of the $J^{P}\left(0^{+}, 1^{+^{\prime}}\right)$ levels are large only for the $D_{s}, B_{s}$ mesons, which lie close to the $D K, D^{*} K, B K, B^{*} K$ thresholds, but not for the $D(1 P), B(1 P)$ mesons, in this way violating symmetry between them (this symmetry is possible in closechannel approximation). In our calculations shifted masses of the $D_{s}\left(0^{+}\right)$and $B_{s}\left(0^{+}\right)$practically coincide with those for the $D\left(0^{+}\right)$and $B\left(0^{+}\right)$, in agreement with the experimental fact that $M_{\exp }\left(D\left(0^{+}\right)\right)=2350 \pm 50 \mathrm{MeV}$ [26] is equal or even larger than $M_{\exp }\left(D_{s}\left(0^{+}\right)\right)=2317$ $\mathrm{MeV}$. The states with $j=3 / 2 D_{s}\left(1^{+}, 2^{+}\right)$and $D\left(1^{+}, 2^{+}\right)$have no mass shifts and for them the mass difference is $\sim 100 \mathrm{MeV}$, that just corresponds to the mass difference between the $s$ and light quark dynamical masses.

For the $D_{s}\left(1^{+^{\prime}}\right)$ and $B_{s}\left(1^{+^{\prime}}\right)$ mesons calculated masses are also close to those of the $D$ and $B$ mesons. Therefore for given chiral dynamics the $J^{P}\left(0^{+}, 1^{+^{\prime}}\right)$ states cannot be considered as the chiral partners of the ground-state multiplet $J^{P}\left(0^{-}, 1^{-}\right)$, as suggested in [11].

We also analyse why two other members of the $1 \mathrm{P}$ multiplet, with $J^{P}=2^{+}$and $1^{+}$, do not acquire the mass shifts due to decay channel coupling (DCC) and have small widths. Such situation occurs if the states $1^{+}$and $1^{+^{\prime}}$ appear to be almost pure $j=\frac{3}{2}$ and $j=\frac{1}{2}$ states. Still small mixing angle between them, $|\phi|<6^{\circ}$, is shown to be compatible with experimental restriction on the width of $D_{s 1}(2536)$, admitting possible admixture of other component in the wave function (w.f.) $\lesssim 10 \%$. 
In our analysis the 4-component (Dirac) structure of the light quark w.f. is crucially important. Specifically, the emission of a NG meson is accompanied with the $\gamma_{5}$ factor which permutes higher and lower components of the Dirac bispinors. For the $j=1 / 2, P$ -wave and the $j=1 / 2, S$-wave states it is exactly the case that this "permuted overlap" of the w.f. is maximal because the lower component of the first state is similar to the higher component of the second state and vice versa. We do not know other examples of such a "fine tuning". On the other hand in the first approximation we neglect an interaction between two mesons in the continuum, like $D K$,etc.

In present paper we concentrate on the $P$-wave $B, B_{s}$ mesons and the effects of the channel coupling. While the $1 \mathrm{P}$ levels of the $D, D_{s}$ mesons are now established with good accuracy [1],[2],[26], for the $B, B_{s}$ mesons only relatively narrow $2^{+}, 1^{+}$states have been recently observed [27], 228]. According to these data the splitting between the $2^{+}$and $1^{+}$ levels is small, $\sim 20-10 \mathrm{MeV}$, while the mass difference between $B_{s}\left(2^{+}\right)$and $B\left(2^{+}\right)$states is again $\sim 100 \mathrm{MeV}$, as for the $D_{s}\left(2^{+}\right)$and $D\left(2^{+}\right)$mesons.

The actual position of the $B(1 P), B_{s}(1 P)$ levels is important for several reasons. Firstly, since dynamics of $(q \bar{b})$ mesons is very similar to that of $q \bar{c}$, the observation of predicted large mass shifts of the $B_{s}\left(0^{+}, 1^{+^{\prime}}\right)$ levels would give a strong argument in favour of the decay channel mechanism suggested here and in [18]. Secondly, observation of all $P$-wave states for the $B, B_{s}$ mesons could clarify many unclear features of spin-orbit and tensor interactions in mesons. Understanding of the decay channel coupling (DCC) mass shifts could become an important step in constructing chiral theory of strong decays with emission of one or several NG particles.

The paper is organized as follows. In the next Section we discuss the formalism from [18], extending that to the case of the $B$, and $B_{s}$ mesons and also to the $1^{+}$states, and discuss the mixing between the $1^{+}$and ${1^{+}}^{\prime}$ states. In Section 3 the masses of HL mesons, calculated in closed-channel approximation, are given. The Section 4 is devoted to the mechanism of chiral transitions while in Section 5 our calculations of the mass shifts due to DCC are presented.The predictions of the $B\left(J^{P}\right), B_{s}\left(J^{P}\right)$ masses and discussion of our results are given in Section 6, while Section 7 contains the Conclusions. In Appendix A a connection between the lowest order of $\Delta L_{F C M}$ and the effective Lagrangian is illustrated. In Appendix $\mathrm{B}$ the details of our calculations of the masses are given, while in Appendix $\mathrm{C}$ the connection between FS splittings and the mixing matrix (and angle) of the $1^{+}$states is discussed. 


\section{MIXING OF THE $1^{+}$AND $1^{+^{\prime}}$ STATES}

It is well known that in single-channel approximation, due to spin-orbit and tensor interactions the $P$-wave multiplet of a HL meson is splitted into four levels with $J^{P}=0^{+}, 1_{L}^{+}, 1_{H}^{+}, 2^{+}$ [29]. Here for the $1^{+}$states we use the notation $\mathrm{H}(\mathrm{L})$ for the higher(lower) eigenstate of the mixing matrix because apriori one cannot say which of them mostly consists of the light quark $j=1 / 2$ contribution (see Appendix C). For a HL meson, strongly coupled to a nearby decay channel (DC),some member(s) of the $P$-wave multiplet can be shifted down while another not.Just such situationis takes place for the $D_{s}(1 P)$ multiplet.

The scheme of classification, more adopted to a HL meson, in the first approximation treats the heavy quark as a static one and therefore the Dirac equation can be used to define the light quark levels and wave functions [10]. Starting with the Dirac's $P$-wave levels, one has the states with $j=1 / 2$ and $j=3 / 2$. Since the light quark momentum $j$ and the quantum number $\varkappa$ are conserved, ${ }^{1}$ they run along the following possible values:

\begin{tabular}{|c|c|c|}
\hline \multicolumn{3}{|c|}{$D$} \\
\hline$J^{P}$ & \begin{tabular}{l|l}
$j$ & $l$
\end{tabular} & $\varkappa$ \\
\hline $0^{-}$ & $\frac{1}{2} \mid 0$ & -1 \\
\hline $1^{-}$ & $\frac{1}{2} \mid 0$ & -1 \\
\hline $1^{-}$ & \begin{tabular}{l|l}
$\frac{3}{2}$ & 2 \\
\end{tabular} & +2 \\
\hline
\end{tabular}

\begin{tabular}{c|c|c|c}
\multicolumn{4}{c}{$D_{s}$} \\
\hline$J^{P}$ & $j$ & $l$ & $\varkappa$ \\
\hline \hline $0^{+}$ & $\frac{1}{2}$ & 1 & +1 \\
\hline $1^{+}$ & $\frac{1}{2}$ & 1 & +1 \\
\hline $1^{+}$ & $\frac{3}{2}$ & 1 & -2 \\
\hline $2^{+}$ & $\frac{3}{2}$ & 1 & -2 \\
\hline \hline $2^{+}$ & $\frac{5}{2}$ & 3 & +3
\end{tabular}

The HL meson w.f. can be expressed in terms of the light quark w.f. - the Dirac bispinors $\psi_{q, s}^{j l M}$ :

$$
\begin{gathered}
\Psi_{D}\left(J_{1 / 2}^{-}, M_{f}\right)=C_{\frac{1}{2}, M_{f}-\frac{1}{2} ; \frac{1}{2},+\frac{1}{2}}^{J, M_{f}} \psi_{q}^{\frac{1}{2}, 0, M_{f}-\frac{1}{2}} \otimes|\bar{c} \uparrow\rangle+C_{\frac{1}{2}, M_{f}+\frac{1}{2} ; \frac{1}{2},-\frac{1}{2}}^{J, M_{f}} \psi_{q}^{\frac{1}{2}, 0, M_{f}+\frac{1}{2}} \otimes|\bar{c} \downarrow\rangle, \\
\Psi_{D_{s}}\left(J_{j}^{+}, M_{i}\right)=C_{j, M_{i}-\frac{1}{2} ; \frac{1}{2},+\frac{1}{2}}^{J, M_{i}} \psi_{s}^{j, 1, M_{i}-\frac{1}{2}} \otimes|\bar{c} \uparrow\rangle+C_{j, M_{i}+\frac{1}{2} ; \frac{1}{2},-\frac{1}{2}}^{J, M_{i}} \psi_{s}^{j, 1, M_{i}+\frac{1}{2}} \otimes|\bar{c} \downarrow\rangle,
\end{gathered}
$$

where $C_{j_{1} M_{1} ; j_{2} M_{2}}^{J M}$ are the corresponding Clebsch-Gordan coefficients.

Later in the w.f. we neglect possible (very small) mixing between $D\left(1_{1 / 2}^{-}\right), D\left(1_{3 / 2}^{-}\right)$states and also between $D_{s}\left(2_{3 / 2}^{+}\right), D_{s}\left(2_{5 / 2}^{+}\right)$states. However, physical $D_{s}\left(1^{+}\right)$states can be mixed

${ }^{1}$ we use here the standard notation $\varkappa=\mp\left|j+\frac{1}{2}\right|$ for $j=\left\{\begin{array}{l}l+\frac{1}{2} \\ l-\frac{1}{2}\end{array}\right.$ 
via open channels and tensor interaction,while the $0^{+}$and $2^{+}$levels are obtained solely from $j=\frac{1}{2}$ and $j=\frac{3}{2}$, respectively.

The eigenstates, defining the higher $1_{H}^{+}$and lower $1_{L}^{+}$levels, can be parametrized by introducing the mixing angle $\phi$ :

$$
\left|1_{H}^{+}\right\rangle=\cos \phi\left|j=\frac{1}{2}\right\rangle+\sin \phi\left|j=\frac{3}{2}\right\rangle
$$

and

$$
\left|1_{L}^{+}\right\rangle=-\sin \phi\left|j=\frac{1}{2}\right\rangle+\cos \phi\left|j=\frac{3}{2}\right\rangle,
$$

where the mixing angle is defined by the unitary mixing matrix $\hat{O}_{\text {mix }}$. In the heavy-quark limit the states with $j=\frac{3}{2}$ and $j=\frac{1}{2}$ are not mixed, but for finite $m_{Q}$ they can be mixed and definition of the mixing matrix in this basis is rather complicate procedure [10], which is also model-dependent. Therefore it is more convenient to connect the angle $\phi$ in (5), (6) with the known factors in the $\mathbf{L S}$ basis, where $\hat{O}_{m i x}$ is well defined in closed-channel approximation and factually depends only on the ratio $a / t$, where $a$ is the spin-orbit and $t$ is the tensor splitting. For our analysis we do not need to know details of spin-orbit interaction (see Appendix C).

Then the splittings of the $2^{+}$and $0^{+}$levels are

$$
\begin{aligned}
& M\left(2^{+}\right)-M_{\operatorname{cog}}=a-0.1 t, \\
& M\left(0^{+}\right)-M_{c o g}=-2 a-t,
\end{aligned}
$$

while $1_{L}^{+}$and $1_{H}^{+}$in (5) and (6) can be expressed through the mixing angle $\theta$ in the expansion of these states in the LS basis, where they represent the decomposition of the ${ }^{3} P_{1}$ and ${ }^{1} P_{1}$ states:

$$
\begin{gathered}
\left|1_{H}^{+}=\cos \theta\right|{ }^{3} P_{1}>-\sin \theta \mid{ }^{1} P_{1}> \\
\left|1_{L}^{+}=\sin \theta\right|^{3} P_{1}>+\cos \theta \mid{ }^{1} P_{1}>.
\end{gathered}
$$

The states ${ }^{3} P_{1}$ and ${ }^{1} P_{1}$ in (8) can be expressed through the basis with the eigenstates $\left|j=\frac{3}{2}\right\rangle$ and $\left.j=\frac{1}{2}\right\rangle$ [29]:

$$
\begin{aligned}
& \left|{ }^{3} P_{1}\right\rangle=\frac{1}{\sqrt{3}}\left|j=\frac{3}{2}\right\rangle+\sqrt{\frac{2}{3}}\left|j=\frac{1}{2}\right\rangle, \\
& \left|{ }^{1} P_{1}\right\rangle=\sqrt{\frac{2}{3}}\left|j=\frac{3}{2}\right\rangle-\frac{1}{\sqrt{3}}\left|j=\frac{1}{2}\right\rangle .
\end{aligned}
$$


TABLE I: The mixing coefficients of the $1^{+}$states for different ratios $a / t$.

\begin{tabular}{|c|c|c|c|}
\hline & $\frac{a}{t}=1.0$ & $\frac{a}{t}=0.950$ & $\frac{a}{t}=0.917$ \\
$\theta=35.264^{\circ}$ & $\theta=43.987^{\circ}$ & $\theta=50.014^{\circ}$ \\
& $\phi=0$ & $\phi=-8.727^{\circ}$ & $\phi=-14.75^{O}$ \\
\hline $1_{H}^{+}$ & $+1.0\left|\frac{1}{2}\right\rangle$ & $-0.152\left|\frac{3}{2}\right\rangle+0.988\left|\frac{1}{2}\right\rangle$ & $-0.255\left|\frac{3}{2}\right\rangle+0.967\left|\frac{1}{2}\right\rangle$ \\
\hline & & & \\
\hline & & & \\
\hline & $1.0\left|\frac{3}{2}\right\rangle$ & $0.988\left|\frac{3}{2}\right\rangle+0.152\left|\frac{1}{2}\right\rangle$ & $0.255\left|\frac{3}{2}\right\rangle+0.967\left|\frac{3}{2}\right\rangle$ \\
\hline
\end{tabular}

Then in closed-channel approximation the following relation can be established between angles $\phi$ and $\theta$ :

$$
\phi=-\theta+35.264^{\circ}
$$

From (10) it follows that

1. If $1_{H}^{+}$is pure $\mid j=\frac{1}{2}>$ state $\left(\phi=0^{\circ}\right)$, then this state is the admixture of the ${ }^{3} P_{1}$ and ${ }^{1} P_{1}$ states with $\theta=35.264^{\circ}$.

2. If $1_{H}^{+}$is pure $\mid j=\frac{3}{2}>$ state $\left(\phi=90^{\circ}\right)$, then in the $\mathbf{L S}$ basis this state is admixture of the ${ }^{3} P_{1}$ and ${ }^{1} P_{1}$ states with $\theta=-54.736^{\circ}$.

3. The special case with $\phi=-9.74^{\circ}$ corresponds to "equal" mixing between the ${ }^{3} P_{1}$ and ${ }^{1} P_{1}$ states with the angle $\theta=-45^{\circ}$.

Therefore the solutions with small $|\phi| \lesssim 6^{\circ}$ correspond to the mixing angle $\theta$ from the range: $29^{\circ} \lesssim \theta \lesssim 41^{\circ}$ for which the ratio $a / t$ appears to be slightly smaller (larger) unity for small negative (positive) $\phi$. Here we consider small negative $\phi$.

The dependence of the mixing angle on the ratio $a / t$ is illustrated by Table $\prod$ taking three different ratios $a / t$.

Thus $1_{H}^{+}$state as the pure $\mid j=\frac{1}{2}>$ corresponds to $a=t$, while for slightly smaller ratio, $a / t=0.95$, the admixture of the $\mid j=\frac{3}{2}>$ state is $\sim 15 \%$ and for $a / t=0.917$ the admixture 
TABLE II: The $B$ meson masses (in $\mathrm{MeV}$ ) (without decay channel coupling)

\begin{tabular}{|c|c|c|c|c|c|c|}
\hline & $0^{-}$ & $1^{-}$ & $0^{+}$ & $1_{L}^{+}$ & $1_{H}^{+}$ & $2^{+}$ \\
\hline $\begin{array}{c}\text { From [20] and } \\
\text { this paper }\end{array}$ & 5279 & 5325 & 5695 & 5726 & 5732 & 5742 \\
\hline experiment & 5279.0 & 5325.0 & & $5721 \pm 5[28]$ & & $5746 \pm 4[28]$ \\
& $\pm 0.5[26]$ & $\pm 0.6[15]$ & & $5725.3_{-3.2}^{+2.4}[29]$ & & $5739.9_{-2.4}^{+2.2}[29]$ \\
\hline
\end{tabular}

is already 26\%. Notice that the physical condition $a \lesssim t$ contradicts the heavy-quark limit when $t \rightarrow 0$ while $a \neq 0$ and can have large magnitude.

The structure of the mixing is important because it defines the order of levels and the value of mass shift for the $1^{+^{\prime}}$ state, as well as the mass shift and the width of another $1^{+}$ level. It is important that if the coupling to nearby continuum channel is taken into account, then as follows from experiment, the position of the $2^{+}$and $1^{+}$levels does not change (within 1-3 MeV) and just their mass difference $\Delta=M\left(2^{+}\right)-M\left(1^{+}\right)$can be used to define tensor splitting: it can be derived that $\Delta=(1.25 \pm 0.15) t$ for any $a / t$.

\section{THE MASSES OF HEAVY-LIGHT MESONS}

In closed-channel approximation the masses of HL mesons, or initial positions of the levels (without channel coupling), can be calculated in different schemes, e.g. in the LS coupling [18], or as in the Dirac type coupling [10]. In Tables [1, III we give these unperturbed masses for the $B$ and $B_{s}$ mesons which are calculated with the use of the relativistic string Hamiltonian [6, 24]. In this approach the $P$-wave masses of HL mesons appear to be smaller that in other potential models because they contain negative string corections (see Appendix B). 
TABLE III: The $B_{s}$ meson masses (in $\mathrm{MeV}$ ) (without decay channel coupling)

\begin{tabular}{|c|c|c|c|c|c|c|}
\hline$J^{P}$ & $0^{-}$ & $1^{-}$ & $0^{+}$ & $1_{L}^{+}$ & $1_{H}^{+}$ & $2^{+}$ \\
\hline This paper & 5362 & 5407 & 5805 & 5830 & 5835 & 5843 \\
and from [20] & & & & & & \\
\hline experiment & 5367.7 & 5411.7 & & 5829.4 & & 5839.1 \\
& $\pm 1.8[26]$ & $\pm 3.2[30]$ & & $\pm 0.8[28]$ & & $\pm 3.0[28]$ \\
& & & & & & \\
\hline
\end{tabular}

Calculated masses of the states with $j=3 / 2,1_{L}^{+}$and $2^{+}$, for the ratio $a / t=0.95$ (see Table II) appear to be in good agreement with recent DO Collab.measurements of the $B$ meson masses [28]. Such agreement can also be reached for other values $a / t$ close to unity:

$$
R=\frac{a}{t}=1.0 \pm 0.05
$$

and we take the same ratio for the $B, B_{s}$ mesons, and also for the $D, D_{s}$ mesons: for such choice the contribution from the $j=\frac{1}{2}$ state dominates in the $1_{H}^{+}$meson. In particular for $a / t=0.95$ :

$$
\left|1_{H}^{+}\right\rangle=0.9884\left|\frac{1}{2}\right\rangle-0.1517\left|\frac{3}{2}\right\rangle
$$

and

$$
\left|1_{L}^{+}\right\rangle=0.1517\left|\frac{1}{2}\right\rangle+0.9884\left|\frac{3}{2}\right\rangle .
$$

The masses given in Tables [I, III are obtained taking the tensor splitting $t \cong 12.2 \mathrm{MeV}$ and $t \cong 10 \mathrm{MeV}$ for the $B$ and $B_{s}$ mesons, respectively. The tensor splittings have been determined to fit the mass difference $M\left(2^{+}\right)-M\left(1^{+}\right)$, which has the important property it does not change (within $2 \mathrm{MeV}$ ) if DCC is taken into account.

In Table IV the masses of the $B, B_{s}$ mesons (from Tables II]III) are compared to those obtained in other models; there the conventional notations $1^{+}$and $1^{+^{\prime}}$ for the $j=\frac{3}{2}$ and 
TABLE IV: Theoretical predictions for the $B(1 P)$ and $B_{s}(1 P)$ masses (in $\mathrm{MeV}$ )(without decay channel coupling)

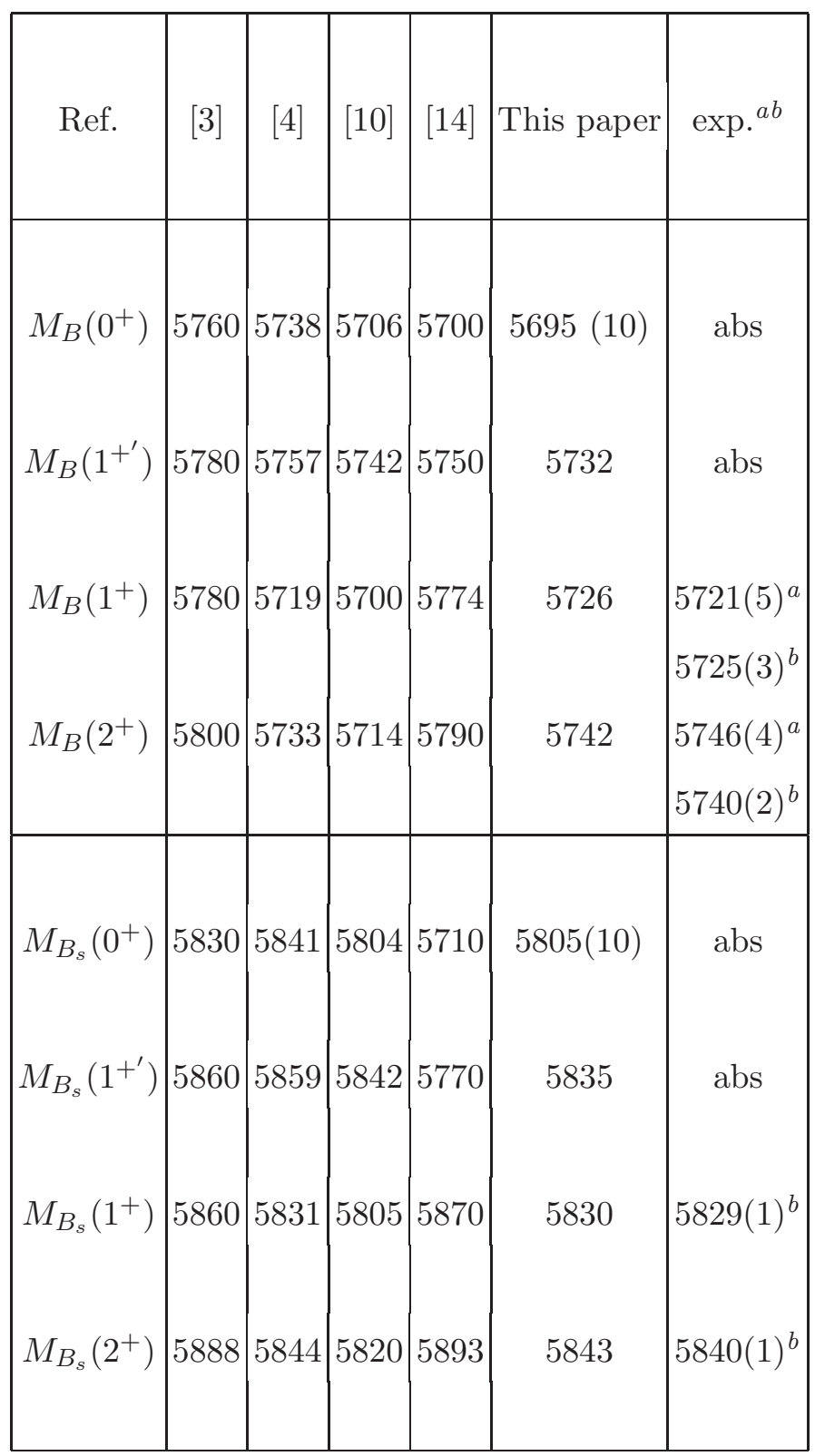

${ }^{a}$ Experimental data of the D0 Collaboration [27]

${ }^{b}$ Experimental data from [28]

$j=\frac{1}{2}$ states are used.

Comparison of the masses,given in TableIV, shows that in different papers $M(B), M\left(B_{s}\right)$ differ not much, within $\pm 50 \mathrm{MeV}$, however, the order of levels inside the 1P multiplet appears 
TABLE V: The masses of the $D(1 P)$ and $D_{s}(1 P)$ mesons in $\mathrm{MeV}$ ) (without decay channel coupling)

\begin{tabular}{|l|l|l|l|l|}
\hline & $0^{+}$ & $1_{L}^{+}$ & $1_{H}^{+}$ & $2^{+}$ \\
\hline$D$ & 2352 & 2423 & 2436 & 2461 \\
\hline experiment & 2350 & 2422 & 2427 & 2459 \\
{$[26]$} & \pm 50 & \pm 1.3 & \pm 51 & \pm 4 \\
\hline$D_{s}$ & 2467 & 2537 & 2550 & 2575 \\
\hline experiment & 2317.3 & 2535.4 & 2459 & 2573.5 \\
\pm 0.6 & \pm 0.8 & \pm 1 & \pm 1.7 \\
\hline
\end{tabular}

to be different.In particular, in [4], [10] the $2^{+}$level has smaller mass than the $1^{+^{\prime}}$ while in our calculations the $2^{+}$state has always maximal mass.It means that FS parameters $a, t$, and their ratio, as well as the mixing angle $\phi$ between the $1^{+}$and $1^{+^{\prime}}$ states, can differ essentially in given papers. Meanwhile existing experimental limit on the width of $D_{s 1}(2536)$ puts strict restrictions on admittable value of the mixing angle $\phi$ (see next Section).

Finally in Table $\mathrm{V}$ we give also unperturbed masses of the $D(1 P)$ and $D_{s}(1 P)$ mesons, taking the splitting $t=29 \mathrm{MeV}$ from the mass difference, $\Delta=M\left(2^{+}\right)-M\left(1^{+}\right)=1.31 t=38$ $\mathrm{MeV}$ and $a / t=0.95$, both for the $D$ and $D_{s}$ mesons.Notice that the position of the $P$-wave mesons does not practically change if $a / t=1.0$ (or $\phi=0)$.

Given in Table $\mathrm{V}$ masses show that in closed-channel approximation we have reached good agreement with experiment for all $D(1 P)$ mesons and also for narrow mesons $D_{s}(2535), D_{s}(2573)$.

We do not need here to know the details of spin-orbit interaction which at present is not fully understood, probably, because of important role of one-loop (or even higher) corrections [31] and possible suppression of NP spin-orbit potential observed on the lattice [32]. Here 
we would like only to notice that in heavy quarkonia the ratios $a / t$ are also close or equal unity:

$$
\begin{gathered}
a / t=1.04 \pm 0.08 \text { for } \chi_{b}(1 P) ; a / t=(1.02 \pm 0.14) \text { for } \chi_{b}(2 P), \\
a / t=(0.86 \pm 0.02) \text { for } \chi_{c}(1 P) .
\end{gathered}
$$

\section{CHIRAL TRANSITIONS}

To obtain the mass shift due to DCC effect we use here the chiral Lagrangian (1), which includes both effects of confinement (embodied in the string tension) and Chiral Symmetry Breaking (CSB) (in Euclidean notations):

$$
L_{F C M}=i \int d^{4} x \psi^{+}(\hat{\partial}+m+\hat{M}) \psi
$$

with the mass operator $\hat{M}$ given as a product of the scalar function $W(r)$ and the $\mathrm{SU}(3)$ flavor octet,

$$
\hat{M}=W(r) \exp \left(i \gamma_{5} \frac{\varphi_{a} \lambda_{a}}{f_{\pi}}\right)
$$

where

$$
\varphi_{a} \lambda_{a}=\sqrt{2}\left(\begin{array}{lll}
\frac{\pi^{0}}{\sqrt{2}}+\frac{\eta^{0}}{\sqrt{6}}, & \pi^{+}, & K^{+} \\
\pi^{-}, & \frac{\eta^{0}}{\sqrt{6}}-\frac{\pi^{0}}{\sqrt{2}}, & K^{0} \\
K^{-}, & \bar{K}^{0}, & -\frac{2 \eta^{0}}{\sqrt{6}}
\end{array}\right) .
$$

Taking the meson emission to the lowest order, one obtains the quark-pion Lagrangian in the form

$$
\Delta L_{F C M}=-\int \psi_{i}^{+}(x) \sigma|\mathbf{x}| \gamma_{5} \frac{\varphi_{a} \lambda_{a}}{f \pi} \psi_{k}(x) d^{4} x
$$

Writing the equation (17) as $\Delta L_{F C M}=-\int V_{i f} d t$, one obtains the operator matrix element for the transition from the light quark state $i$ (i.e. the initial state $i$ of a HL meson) to the continuum state $f$ with the emission of a NG meson $\left(\varphi_{a} \lambda_{a}\right)$. Thus we are now able to write the coupled channel equations, connecting any state of a HL meson to a decay channel which contains another HL meson plus a NG meson.

In the case, when interaction in each channel and also in the transition operator is timeindependent, one can write following system of equations (see [33] for a review)

$$
\left[\left(H_{i}-E\right) \delta_{i l}+V_{i l}\right] G_{l f}=1
$$


Such two-channel system of the equations can be reduced to one equation with additional DCC potential, or the Feshbach potential [34],

$$
\left(H_{1}-E\right) G_{11}-V_{12} \frac{1}{H_{2}-E} V_{21} G_{11}=1 .
$$

Considering a complete set of the states $|f\rangle$ in the decay channel 2 and the set of unperturbed states $|i\rangle$ in channel 1 , one arrives at the nonlinear equation for the shifted mass $E$,

$$
E=E_{1}^{(i)}-\sum_{f}\left\langle i\left|V_{12}\right| f\right\rangle \frac{1}{E_{2}^{(f)}-E}\left\langle f\left|V_{21}\right| i\right\rangle .
$$

Here the unperturbed values of $E_{1}^{(i)}$ are assumed to be known (see Tables II, III, V]), while the interaction $U_{i f}$ is defined in (17). A solution of the nonlinear equation (20) yields (in general a complex number $E=\bar{E}-\frac{i \Gamma}{2}$ ) one or more roots on all Riemann sheets of the complex mass plane.

\section{CALCULATION OF THE DCC SHIFTS}

To calculate explicitly the mass shifts, we will use the Eq. (20) in the following form:

$$
m[i]=m^{(0)}[i]-\sum_{f} \frac{|<i| \hat{V}|f>|^{2}}{E_{f}-m[i]},
$$

where $m^{(0)}[i]$ is the initial mass, $m[i]$ - is the final one, $E_{f}=\omega_{D}+\omega_{K}$ is the energy of the final state, and the operator $\hat{V}$ provides the transitions between the channels (see the comment after Eq. (17)).

In our approximation we do not take into account the final state interaction in the $D K$ system and neglect the $D$-meson motion, so the w.f. of the $i, f$-states are:

$$
\left|f>=\Psi_{K}(\mathbf{p}) \otimes \Psi_{D}\left(M_{f}\right), \quad\right| i>=\Psi_{D_{s}}\left(M_{i}\right),
$$

where

$$
\Psi_{K}(\mathbf{p})=\frac{e^{i \mathbf{p r}}}{\sqrt{2 \omega_{K}(\mathbf{p})}}
$$

is the plane wave describing the $K$-meson and $\Psi_{D}\left(M_{f}\right), \Psi_{D_{s}}\left(M_{i}\right)$ are the HL meson w.f. at rest with the spin projections $M_{f}, M_{i}$, respectively.

We introduce the following notations:

$$
\omega_{K}(\mathbf{p})=\sqrt{\mathbf{p}^{2}+m_{K}^{2}}, \quad \omega_{D}(\mathbf{p})=\sqrt{\mathbf{p}^{2}+m_{D}^{2}},
$$


so that in the final state the total energy is $E_{f}=\omega_{D}+\omega_{K}$, while

$$
T_{f}=E_{f}-m_{D}-m_{K}
$$

is the kinetic energy. Also it is convenient to define other masses with respect to nearby threshold: $m_{t h r}=m_{K}+m_{D}$,

$$
\begin{gathered}
E_{0}=m^{(0)}\left[D_{s}\right]-m_{D}-m_{K}, \quad \delta m=m\left[D_{s}\right]-m^{(0)}\left[D_{s}\right], \\
\Delta=E_{0}+\delta m=m\left[D_{s}\right]-m_{D}-m_{K},
\end{gathered}
$$

where $\Delta$ determines the deviation of the $D_{s}$ meson mass from the threshold. In what follows we consider unperturbed masses $m_{0}\left(J^{P}\right)$ of the $(Q \bar{q})$ levels as given (our results do not change if we slightly vary their position, in this way the analysis is actually model-independent).

Using these notations, the Eq.(20) can be rewritten as

$$
E_{0}-\Delta=\mathcal{F}(\Delta)
$$

where

$$
\mathcal{F}(\Delta) \stackrel{\text { def }}{=} \int \frac{d^{3} \mathbf{p}}{(2 \pi)^{3}} \sum_{M_{f}} \frac{\left|\left\langle M_{i}|\hat{V}| \mathbf{p}, M_{f}\right\rangle\right|^{2}}{T_{f}(\mathbf{p})-\Delta}
$$

and

$$
\left\langle M_{i}|\hat{V}| \mathbf{p}, M_{f}\right\rangle=-\int \Psi_{D_{s}}^{\dagger}\left(M_{i}\right) \sigma|\mathbf{r}| \gamma_{5} \frac{\sqrt{2}}{f_{K}} \Psi_{D}\left(M_{f}\right) \frac{e^{i \mathbf{p r}}}{\sqrt{2 \omega_{K}(\mathbf{p})}} d^{3} \mathbf{r},
$$

The function $\mathcal{F}(\Delta)$ for negative $\Delta$ diminishes monotonously so there exists a final (critical) point,

$$
E_{0}^{\mathrm{crit}}=\mathcal{F}(-0)
$$

Thus, while solving the Eq.(28), one has two possible situations: $E_{0}<E_{0}^{\text {crit }}$ and $E_{0}>E_{0}^{\text {crit }}$.

In the first case Eq.(28) has a negative real root $\Delta<0$ (see Fig. 1) and the resulting mass of the $D_{s}$ meson appears to be under the threshold. In the second case Eq.(28) has a complex root $\Delta=\Delta^{\prime}+i \Delta^{\prime \prime}$ with positive real part $\Delta^{\prime}>0$ (see Fig. 2) and negative imaginary part $\Delta^{\prime \prime}<0$. To find latter solutions one should make analytic continuation of the solution(s) from the upper halfplane of $\Delta$ under the cut, which starts at the threshold, to the lower halfplane (second sheet). This solution can be also obtained by deforming the integration contour in $T_{f}(p)$. In actual calculations we take infinitesimal imaginary part $\Delta^{\prime \prime}$, proving that $\Delta$ does not change much for finite $\Delta^{\prime \prime}$ (the similar procedure has been used in 
FIG. 1: Eq.(28) for the case $E_{0}<E_{0}^{\text {crit }}$

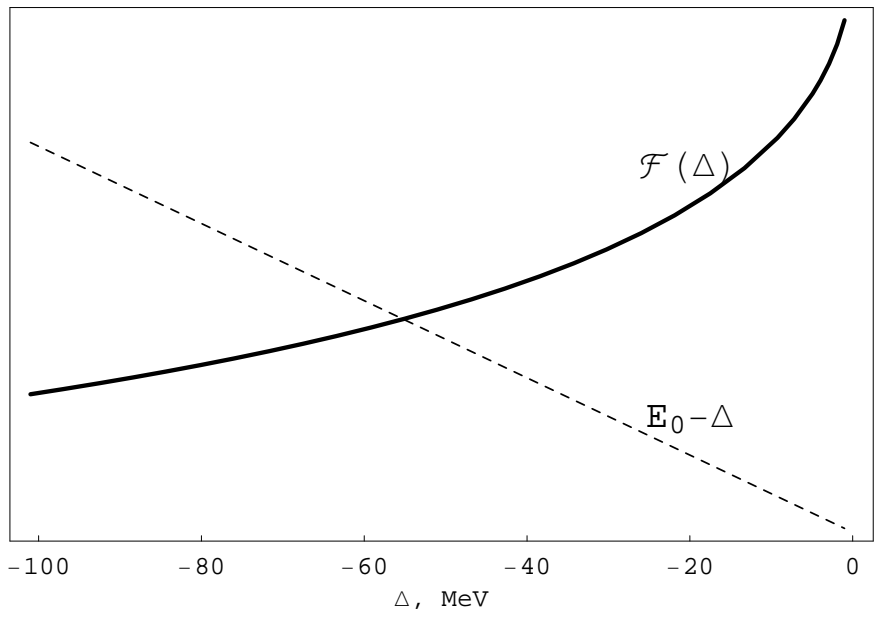

FIG. 2: Eq.(28) for the case $E_{0}>E_{0}^{\text {crit }}$

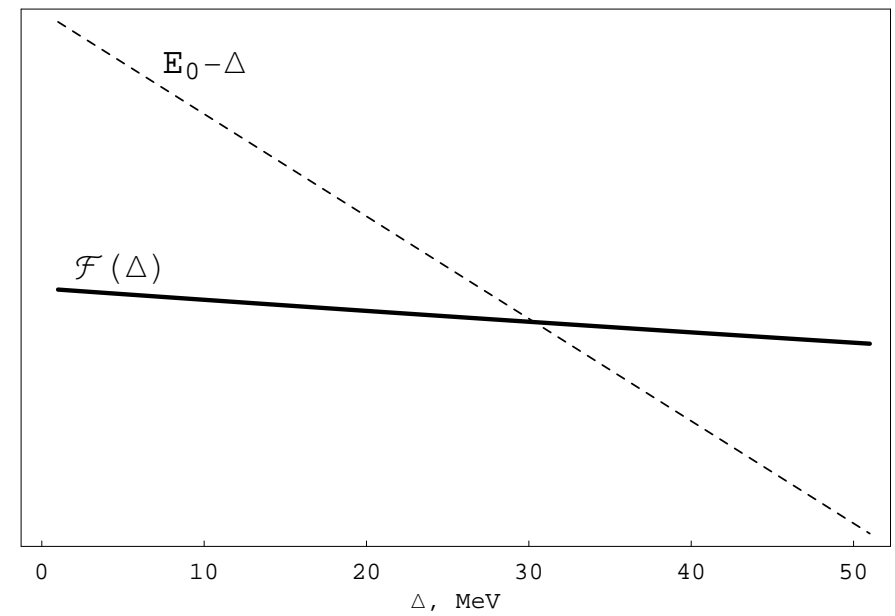

[18]). Finally, the resulting mass of the $D_{s}$ meson proves to be in the complex plane at the position $\Delta^{\prime}-i\left|\Delta^{\prime \prime}\right|$, i.e. the meson has the finite width $\Gamma=2 \Delta^{\prime \prime}$.

For further calculations we should insert the explicit meson w.f. to the matrix element (30). As discussed above, in a HL meson we consider a light quark $q$ moving in the static field of a heavy antiquark $\bar{Q}$, and therefore its w.f. can be taken as the Dirac bispinor:

$$
\psi_{q}^{j l M}=\left(\begin{array}{c}
g(r) \Omega_{j l M} \\
(-1)^{\frac{1+l-l^{\prime}}{2}} f(r) \Omega_{j l^{\prime} M}
\end{array}\right), \quad \int_{0}^{\infty}\left(f^{2}+g^{2}\right) r^{2} d r=1,
$$

where the functions $g(r)$ and $f(r)$ are the solutions of the Dirac equation:

$$
\begin{aligned}
& g^{\prime}+\frac{1+\varkappa}{r} g-\left(E_{q}+m_{q}+U-V_{C}\right) f=0, \\
& f^{\prime}+\frac{1-\varkappa}{r} f+\left(E_{q}-m_{q}-U-V_{C}\right) g=0 .
\end{aligned}
$$


Here the interaction between the quark and the antiquark is described by a sum of linear scalar potential and the vector Coulomb potential with $\alpha_{s}=$ const:

$$
U=\sigma r, \quad V_{C}=-\frac{\beta}{r}, \quad \beta=\frac{4}{3} \alpha_{s} .
$$

Introducing new dimensionless variables

$$
x=r \sqrt{\sigma}, \quad \varepsilon_{q}=E_{q} / \sqrt{\sigma}, \quad \mu_{q}=m_{q} / \sqrt{\sigma},
$$

and new dimensionless functions

$$
g=\sigma^{3 / 4} \frac{G(x)}{x}, \quad f=\sigma^{3 / 4} \frac{F(x)}{x}, \quad \int_{0}^{\infty}\left(F^{2}+G^{2}\right) d x=1,
$$

we come to the following system of equations:

$$
\begin{aligned}
& G^{\prime}+\frac{\varkappa}{x} G-\left(\varepsilon_{q}+\mu_{q}+x+\frac{\beta}{x}\right) F=0, \\
& F^{\prime}-\frac{\varkappa}{x} F+\left(\varepsilon_{q}-\mu_{q}-x+\frac{\beta}{x}\right) G=0 .
\end{aligned}
$$

This system has been solved numerically.

Using the parameters from the papers [18], [39]:

$$
\begin{aligned}
\sigma & =0.18 \mathrm{GeV}^{2}, \quad \alpha_{s}=0.39 \\
m_{s} & =210 \mathrm{MeV}, \quad m_{q}=4 \mathrm{MeV},
\end{aligned}
$$

we obtain the following Dirac eigenvalues $\varepsilon$ :

\begin{tabular}{r|l|l}
$\varkappa$ & $\bar{Q} q, \mu_{q}=0.01$ & $\bar{Q} s, \mu_{s}=0.5$ \\
\hline \hline-1 & 1.0026 & 1.28944 \\
\hline+1 & 1.7829 & 2.08607 \\
\hline-2 & 1.7545 & 2.08475
\end{tabular}

and corresponding eigenfunctions $G, F$ are given in Figs. 3, 4).

Later we use the simplified notations for the quark bispinors:

$$
\psi_{1}\left(M_{1}\right) \stackrel{\text { def }}{=} \psi_{s}^{\frac{1}{2}, 1, M_{1}}, \quad \psi_{2}\left(M_{2}\right) \stackrel{\text { def }}{=} \psi_{q}^{\frac{1}{2}, 0, M_{2}}, \quad \psi_{3}\left(M_{3}\right) \stackrel{\text { def }}{=} \psi_{s}^{\frac{3}{2}, 1, M_{3}} .
$$

Now, using explicit expressions for the spherical spinors,

$$
\Omega_{l+1 / 2, l, M}=\left[\begin{array}{c}
\sqrt{\frac{j+M}{2 j}} Y_{l, M-1 / 2} \\
\sqrt{\frac{j-M}{2 j}} Y_{l, M+1 / 2}
\end{array}\right], \quad \Omega_{l-1 / 2, l, M}=\left[\begin{array}{c}
-\sqrt{\frac{j-M+1}{2 j+2}} Y_{l, M-1 / 2} \\
\sqrt{\frac{j+M+1}{2 j+2}} Y_{l, M+1 / 2}
\end{array}\right],
$$




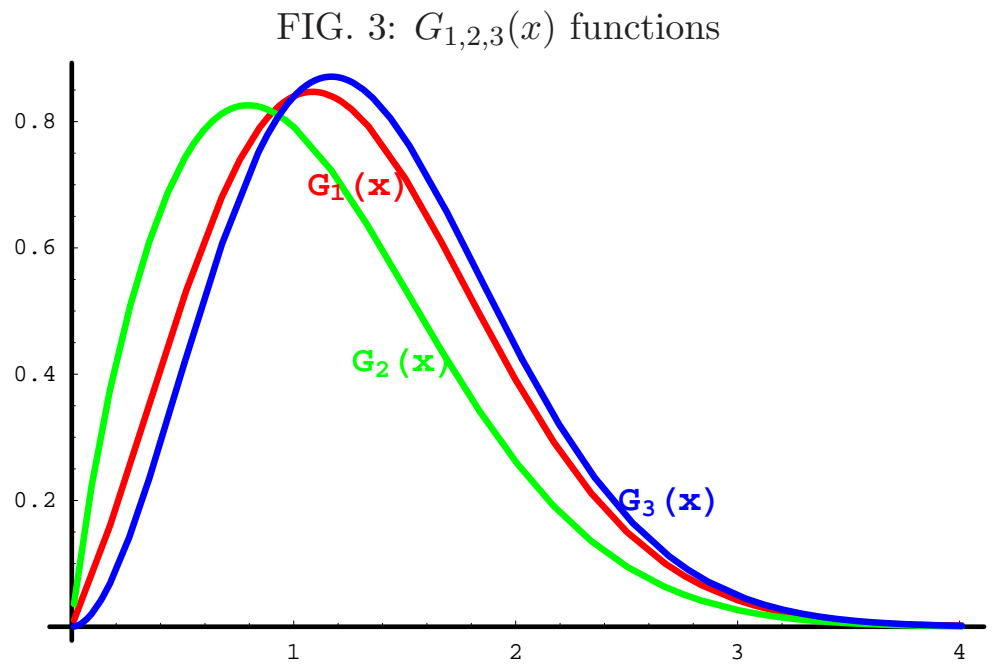

FIG. 4: $F_{1,2,3}(x)$ functions

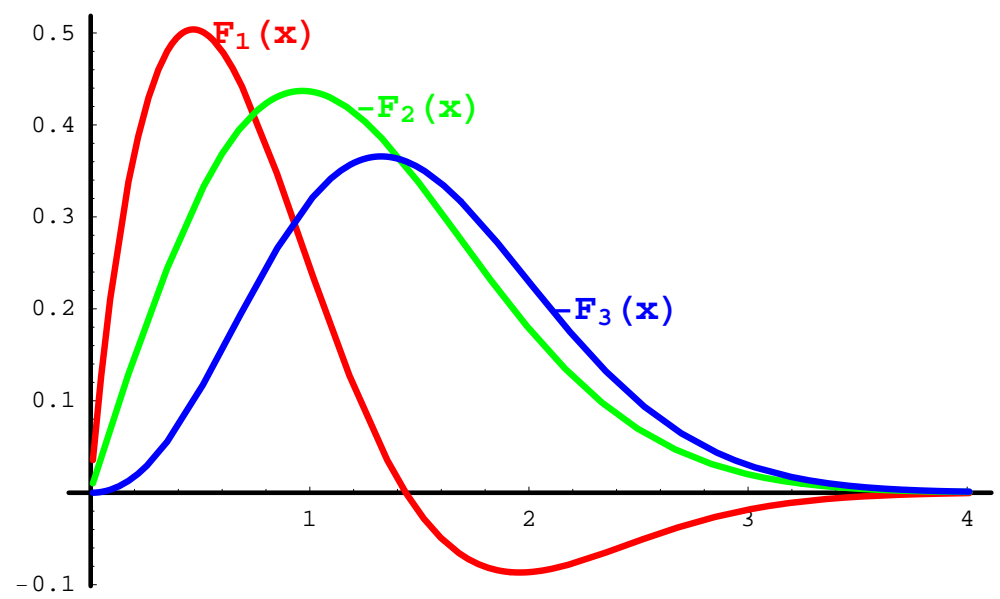

and the expansion :

$$
e^{i \mathbf{p r}}=4 \pi \sum_{l, M} i^{l} j_{l}(p r) Y_{l, M}^{*}\left(\frac{\mathbf{p}}{p}\right) Y_{l, M}\left(\frac{\mathbf{r}}{r}\right),
$$

after cumbersome transformations (which are omitted in the text) we obtain the transition matrix elements:

$$
\begin{aligned}
\left\|\mathcal{V}_{12}\right\|_{M_{1}, M_{2}}=-\int \psi_{1}^{\dagger}\left(M_{1}\right) \sigma|\mathbf{r}| \gamma_{5} \frac{\sqrt{2}}{f_{K}} \psi_{2}\left(M_{2}\right) \frac{e^{i \mathbf{p r}}}{\sqrt{2 \omega_{K}(\mathbf{p})}} d^{3} \mathbf{r}= \\
=\frac{\sqrt{\sigma}}{f_{K} \sqrt{\omega_{K}(p)}} \Phi_{0}\left(\frac{p}{\sqrt{\sigma}}\right) \sqrt{4 \pi} Y_{0, M_{1}-M_{2}}^{*}\left(\frac{\mathbf{p}}{p}\right)
\end{aligned}
$$


FIG. 5: $\Phi_{0,2}(q)$ functions

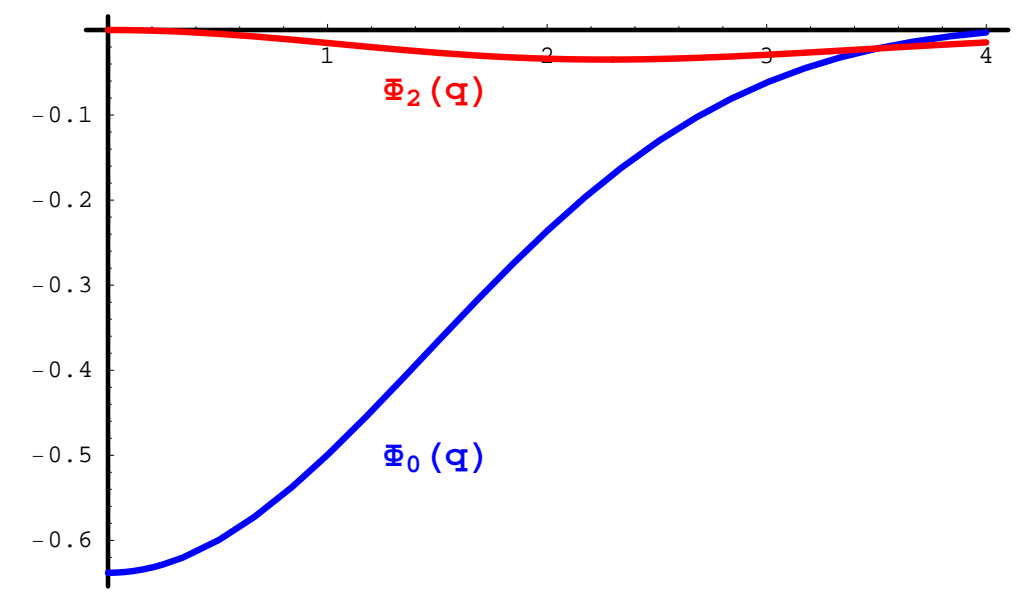

$$
\begin{aligned}
& \left\|\mathcal{V}_{32}\right\|_{M_{3}, M_{2}}=-\int \psi_{3}^{\dagger}\left(M_{3}\right) \sigma|\mathbf{r}| \gamma_{5} \frac{\sqrt{2}}{f_{K}} \psi_{2}\left(M_{2}\right) \frac{e^{i \mathbf{p r}}}{\sqrt{2 \omega_{K}(\mathbf{p})}} d^{3} \mathbf{r}= \\
& =-\frac{\sqrt{\sigma}}{f_{K} \sqrt{\omega_{K}(p)}} \Phi_{2}\left(\frac{p}{\sqrt{\sigma}}\right) \sqrt{\frac{4 \pi}{5}} Y_{2, M_{3}-M_{2}}^{*}\left(\frac{\mathbf{p}}{p}\right) \cdot\left[\begin{array}{cc}
-1 & +2 \\
-\sqrt{2} & +\sqrt{3} \\
-\sqrt{3} & +\sqrt{2} \\
-2 & +1
\end{array}\right] .
\end{aligned}
$$

where

$$
\begin{aligned}
& \Phi_{0}(q)=\int_{0}^{\infty} j_{0}(q x) x d x\left[G_{1}(x) F_{2}(x)-F_{1}(x) G_{2}(x)\right], \\
& \Phi_{2}(q)=\int_{0}^{\infty} j_{2}(q x) x d x\left[G_{3}(x) F_{2}(x)-F_{3}(x) G_{2}(x)\right] .
\end{aligned}
$$

Notice that because of different signs of the $F_{1}(x)$ and $F_{2,3}(x)$ functions (while the $G_{1,2,3}$ functions are all positive) on almost all real axis, the integral $\Phi_{2}$ appears to be strongly suppressed in comparison with the integral $\Phi_{0}$. This fact is confirmed by numerical simulations (see Fig. 5).

Finally, introducing universal functions

$$
\begin{gathered}
\tilde{\mathcal{F}}_{0,2}(\Delta)=\frac{\sigma}{2 \pi^{2} f_{K}^{2}} \int_{0}^{\infty} \frac{p\left(T_{f}\right) \omega_{D}\left(T_{f}\right) d T_{f}}{T_{f}+m_{D}+m_{K}} \cdot \frac{\Phi_{0,2}^{2}\left(\frac{p\left(T_{f}\right)}{\sqrt{\sigma}}\right)}{T_{f}-\Delta}, \\
\tilde{\Gamma}_{0,2}\left(T_{f}\right)=\frac{\sigma}{\pi f_{K}^{2}} \cdot \frac{p\left(T_{f}\right) \omega_{D}\left(T_{f}\right)}{T_{f}+m_{D}+m_{K}} \cdot \Phi_{0,2}^{2}\left(\frac{p\left(T_{f}\right)}{\sqrt{\sigma}}\right)
\end{gathered}
$$


we come to the following equations to determine meson masses and widths:

$$
\begin{array}{c||l}
D_{s}\left(0^{+}\right) & E_{0}\left[0^{+}\right]-\Delta=\tilde{\mathcal{F}}_{0}(\Delta), \\
D_{s}\left(1_{L}^{+}\right) & E_{0}\left[1_{L}^{+}\right]-\Delta=\cos ^{2} \phi \cdot \tilde{\mathcal{F}}_{0}(\Delta)+\sin ^{2} \phi \cdot \tilde{\mathcal{F}}_{2}(\Delta), \\
D_{s}\left(1_{H}^{+}\right) & E_{0}\left[1_{H}^{-}\right]-\Delta^{\prime}=\sin ^{2} \phi \cdot \tilde{\mathcal{F}}_{0}\left(\Delta^{\prime}\right)+\cos ^{2} \phi \cdot \tilde{\mathcal{F}}_{2}\left(\Delta^{\prime}\right), \\
& \Gamma\left[1_{H}^{+}\right]=\sin ^{2} \phi \cdot \tilde{\Gamma}_{0}\left(\Delta^{\prime}\right)+\cos ^{2} \phi \cdot \tilde{\Gamma}_{2}\left(\Delta^{\prime}\right), \\
D_{s}\left(2_{3 / 2}^{+}\right) & E_{0}\left[2_{3 / 2}^{+}\right]-\Delta^{\prime}=\frac{3}{5} \cdot \tilde{\mathcal{F}}_{2}\left(\Delta^{\prime}\right), \\
& \Gamma\left[2_{3 / 2}^{+}\right]=\frac{3}{5} \cdot \tilde{\Gamma}_{2}\left(\Delta^{\prime}\right) .
\end{array}
$$

\section{RESULTS AND DISCUSSION}

In this chapter, using the expressions (47) to define the $D_{s}$ and $B_{s}$ meson mass shifts, we present and discuss our results. We will take into account the following pairs of mesons in coupled channels ( $i$ refers to first (initial)channel, while $f$ refers to second (decay) one):

\begin{tabular}{c||c|c|c}
$i$ & $D_{s}\left(0^{+}\right)$ & $D_{s}\left(1^{+}\right)$ & $D_{s}\left(2^{+}\right)$ \\
\hline$f$ & $D\left(0^{-}\right)+K\left(0^{-}\right)$ & $D^{*}\left(1^{-}\right)+K\left(0^{-}\right)$ & $D^{*}\left(1^{-}\right)+K\left(0^{-}\right)$ \\
$i$ & $B_{s}\left(0^{+}\right)$ & $B_{s}\left(1^{+}\right)$ & $B_{s}\left(2^{+}\right)$ \\
\hline$f$ & $B\left(0^{-}\right)+K\left(0^{-}\right)$ & $B^{*}\left(1^{-}\right)+K\left(0^{-}\right)$ & $B^{*}\left(1^{-}\right)+K\left(0^{-}\right)$
\end{tabular}

In our calculations we use here the following meson masses and thresholds (in $\mathrm{MeV}$ ):

$$
\begin{aligned}
& m_{D^{+}}=1869, \quad m_{D^{+}}+m_{K^{-}}=2363, \\
& m_{D^{*+}}=2010, \quad m_{D^{*+}}+m_{K^{-}}=2504, \\
& m_{B^{+}}=5279, \quad m_{B^{+}}+m_{K^{-}}=5772, \\
& m_{B^{*}}=5325, \quad m_{B^{*}}+m_{K^{-}}=5819 .
\end{aligned}
$$

The results of our calculation are presented in Tables VIVIII. A priori one cannot say whether the $\left|j=\frac{1}{2}\right\rangle$ and $\left|j=\frac{3}{2}\right\rangle$ states are mixed or not.In [35] in the case when there is no mixing at all, the width $\Gamma\left(D_{s 1}(2536)\right)=0.3 \mathrm{MeV}$ is obtained, while now the experimental limit is $\Gamma<2.3 \mathrm{MeV}$ [26] and recently in [36] the width $\Gamma=1.0 \pm 0.17 \mathrm{MeV}$ has been measured. Therefore small mixing is not excluded and here we take the mixing angle $\phi$ slightly deviated from $\phi=0^{\circ}$ (when there is no mixing at all). Then one can define those 
TABLE VI: $D_{s}\left(0^{+}\right)$-meson mass shift due to the $D K$ decay channel and $B_{s}\left(0^{+}\right)$-meson mass shift due to the $B K$ decay channel (all in $\mathrm{MeV}$ )

\begin{tabular}{|c|c|c|c|c|}
\hline state & $m^{(0)}$ & $m^{\text {(theor) }}$ & $m^{(\exp )}$ & $\delta m$ \\
\hline \hline$D_{s}\left(0^{+}\right)$ & 2467 & 2331 & 2317 & -136 \\
\hline$B_{s}\left(0^{+}\right)$ & 5805 & 5700 & not seen & -105 \\
\hline
\end{tabular}

TABLE VII: The $D_{s}\left(1^{+}\right), D_{s}\left(2^{+}\right)$meson mass shifts and widths due to the $D^{*} K$ decay channel for the mixing angle $4^{\circ}$ (all in $\mathrm{MeV}$ )

\begin{tabular}{|c|c|c|c|c|c|c|}
\hline state & $m^{(0)}$ & $m^{\text {(theor })}$ & $m^{(\exp )}$ & $\Gamma_{\left(D^{*} K\right)}^{(\text {theor })}$ & $\Gamma_{\left(D^{*} K\right)}^{(\exp )}$ & $\delta m$ \\
\hline \hline$D_{s}\left(1_{H}^{+}\right)$ & 2550 & 2440 & 2460 & $\times$ & $\times$ & -110 \\
\hline$D_{s}\left(1_{L}^{+}\right)$ & 2537 & 2535 & 2535 & 1.1 & $<1.3$ & -2 \\
\hline$D_{s}\left(2_{3 / 2}^{+}\right)$ & 2575 & 2573 & 2573 & 0.03 & not seen & -2 \\
\hline
\end{tabular}

angles $\phi$ which are compatible with experimental data for the masses and widths of both $1^{+}$ states. The limiting angle $|\phi|=5.7^{\circ}$ (given in Table IX] corresponds to the mixing between the ${ }^{3} P_{1}$ and ${ }^{1} P_{1}$ with $\theta=41^{\circ}$ in $\mathbf{L S}$ scheme.

The value $\xi=(0.995)^{2}$ for the $1_{H}^{+}$states provides large mass shift $(\sim 100 \mathrm{MeV})$ of this level and at the same time does not produce the mass shift of the $1_{L}^{+}$level, which is almost pure $j=\frac{3}{2}$ state. For illustration we show the scheme of the $1^{+}, 2^{+}$shifts on Figures 6 , 7 , We would like to stress here that the dependence of the shift on the heavy non-strange meson

TABLE VIII: The $B_{s}\left(1^{+}\right), B_{s}\left(2^{+}\right)$meson mass shifts and widths due to the $B^{*} K$ decay channel for the mixing angle $4^{\circ}$ (all in $\mathrm{MeV}$ )

\begin{tabular}{|c|c|c|c|c|c|c|}
\hline state & $m^{(0)}$ & $m^{\text {(theor) }}$ & $m^{(\exp )}$ & $\Gamma_{\left(B^{*} K\right)}^{(\text {theor })}$ & $\Gamma_{\left(B^{*} K\right)}^{(\exp )}$ & $\delta m$ \\
\hline \hline$B_{s}\left(1_{H}^{+}\right)$ & 5835 & 5727 & not seen & $\times$ & $\times$ & -108 \\
\hline$B_{s}\left(1_{L}^{+}\right)$ & 5830 & 5828 & 5829 & 0.8 & $<2.3$ & -2 \\
\hline$B_{s}\left(2_{3 / 2}^{+}\right)$ & 5842 & 5840 & 5840 & $<10^{-3}$ & not seen & -2 \\
\hline
\end{tabular}


TABLE IX: The mixing coefficients in Eq.(20) for the DCC shifts of the $B_{s}, D_{s}$ mesons $\left(\phi=5.7^{\circ}\right)$.

\begin{tabular}{|c|c|c|c|}
\hline & & $1_{H}^{+}$ & $1_{L}^{+}$ \\
& $a / t$ & $\cos ^{2} \phi$ & $\sin ^{2} \phi$ \\
\hline$D_{s}(1 P), B_{s}(1 P)$ & 0.96 & $(0.995)^{2}$ & $(0.100)^{2}$ \\
\hline$D_{s}(1 P), B_{s}(1 P)$ & 1.0 & 1.0 & 0 \\
& & & \\
\hline
\end{tabular}

FIG. 6: Scheme of $D_{s}\left(1^{+}, 2^{+}\right)$shifts due to chiral coupling

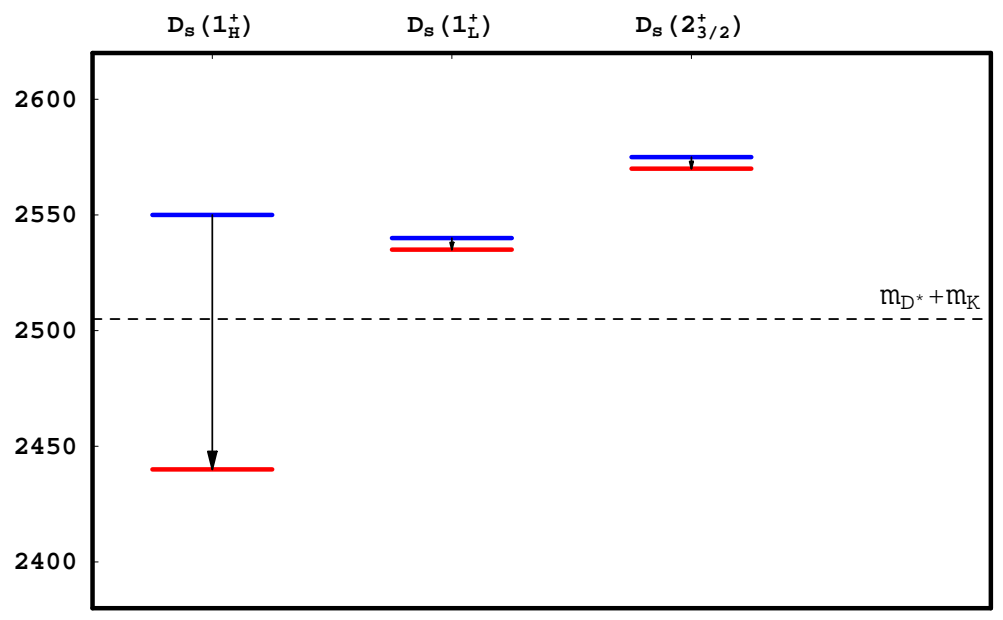

mass (or, equivalently, on the heavy quark mass) is rather weak, which follows directly from the Eq. (47) via inverse mass expansion.

If one uses one-gluon exchange potential for tensor interaction, then to obtain the splitting $t \sim 12 \mathrm{MeV}$ for the $B$ and $B_{s}$ mesons and $t=29 \mathrm{MeV}$ for the $D$ and $D_{s}$ mesons one needs to take $\alpha_{s}\left(\mu_{F S}\right) \sim 0.39$ for all $P$-wave $H L$ mesons. However, in OGE approximation the spin-orbit splitting does not satisfy the condition $a=0.95 t$ for this value of $\alpha_{s}$. This fact possibly indicates on important role of one-loop or even higher radiative corrections, observed in heavy quarkonia [3], and also on possible $\sim 20 \%$ suppression of NP spin-orbit confining potential observed on the lattice [32]. 
FIG. 7: Scheme of $B_{s}\left(1^{+}, 2^{+}\right)$shifts due to chiral coupling

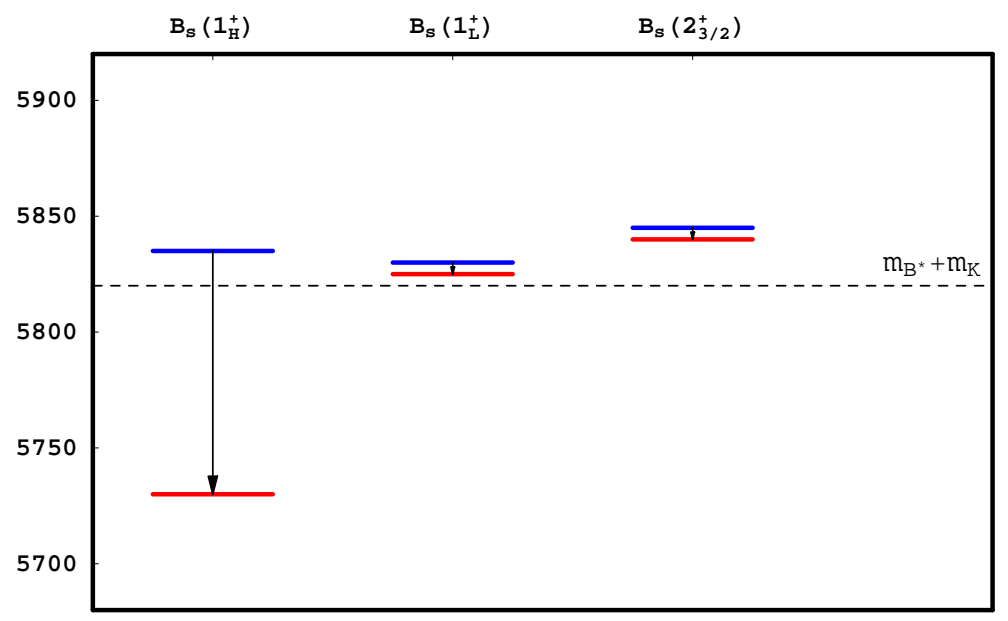

\section{CONCLUSIONS}

We have studied the mass shifts of the $D_{s}\left(0^{+}, 1^{+^{\prime}}\right)$ and $B_{s}\left(0^{+}, 1^{+^{\prime}}\right)$ mesons due to strong coupling to the decay channels $D K, D^{*} K$ and $B K, B^{*} K$. To this end the chiral quark-pion Lagrangian without fitting parameters has been used.

We have shown that the emission of a NG meson, accompanied with the $\gamma_{5}$ factor, gives rise to maximal overlapping between the higher component with $j=\frac{1}{2}$ of the $P$-wave meson $\left(D_{s}, B_{s}\right)$ bispinor w.f. and the lower component (also with $j=\frac{1}{2}$ ) of the $S$-wave meson w.f. in considered $S$-wave decay channel. Due to this effect, while taking the w.f. of the $1 P$ and $1 S$ states with the use of the Dirac equation, large mass shifts of the $0^{+}, 1^{+^{\prime}}$ states are obtained.

The widths of $D_{s 1}(2536)$ and $B_{s 1}(5830)$ are also calculated. To satisfy the experimental condition $\Gamma\left(D_{s 1}(2536)\right)<2.3 \mathrm{MeV}$ the following limit on the mixing angle $\phi$ (between the $\mid j=\frac{3}{2}>$ and $\mid j=\frac{1}{2}>$ states $)$ is obtained, $|\phi| \lesssim 6^{\circ}$. This restriction implies that the mixing angle $\theta$ between the ${ }^{3} P_{1}$ and ${ }^{1} P_{1}$ states in LS basis lies in the range: $29^{\circ} \lesssim \theta \lesssim 41^{\circ}$. For given angles $\theta$ the ratio of the spin-orbit and tensor splittings appears to be close to unity $a / t=1.0 \pm 0.05$, as it takes place for the $\chi_{b}$ and $\chi_{c}$ mesons. The value of tensor splitting $t$ has been defined from the mass difference, $M\left(2^{+}\right)-M\left(1^{+}\right)$, which is not affected by the coupling to the decay channel.

Calculated masses of the $2^{+}$and $1^{+}$states are in good agreement with experiment for all $D, D_{s}, B, B_{s}$ mesons.

For the $0^{+}, 1^{+^{\prime}}$ states, taking into account the mass shifts, the following masses are 
predicted:

- $M\left(B_{s}^{*}\right)=5695(10) \mathrm{MeV}$ which coincides with $M\left(B^{*}\right)=5695(10) \mathrm{MeV}$,

- $M\left(B_{s}\left(1^{+^{\prime}}\right)\right)=5730(10) \mathrm{MeV}$, close to $M\left(B_{1}\left(1^{+^{\prime}}\right)\right)=5732(10) \mathrm{MeV}$.

\section{Acknowledgments}

The authors would like to thank for the support the President Grant for scientific schools

\# 843.2006.2. One of the authors (M.A.T.) thanks RFBR for the partial support via grant \# 06-02-17120.

[1] B.Aubert et al. (Babar Collab.), Phys. Rev. Lett. 90, 242001 (2003).

[2] D.Besson et al. (CLEO Collab), Phys. Rev. D 68, 032002 (2003); P.Krokovny et al. (BelleCollab), Phys. Rev. Lett. 91, 262002 (2003).

[3] S.Godfrey, N.Isgur, Phys. Rev. D 32, 189 (1985); S.Godfrey, R.Kokoski, Phys. Rev. D 43, 1679 (1991).

[4] D.Ebert, V.O.Galkin, R.N.Faustov, Phys. Rev. D 57, 5663 (1998) [Erratum: ibid. D 59, 019902 (1999)].

[5] S.Godfrey, Phys.Rev. D 72, 054029 (2005).

[6] Yu.S.Kalashnikova, A.V.Nefediev, Yu.A.Simonov, Phys. Rev. D 64, 014037 (2001); Yu.S.Kalashnikova, A.V. Nefediev, Phys. Lett. B 492, 91 (2000).

[7] R.Lewis, R.M.Woloshyn, Phys. Rev. D 62, 114507 (2000);

G.S.Bali, Phys.Rev. D 68, 0715001 (2003);

A.Gougall et. al , Phys. Lett. B 569, 4 (3003).

[8] Z.G.Wang, S.L.Wan, Phys. Rev. D 73, 094020 (2006); Z.G.Wang, J.Phys. G 34, 753 (2007).

[9] S.Narison, Phys. Lett. B 605, 319 (2005);

Y.B.Dai, C.S.Huang, C.Liu, S.L. Zhu, Phys. Rev. D 68 , 114011 (2003).

[10] M.Di Pierro, E.J Eichten, Phys. Rev. D 64, 114004 (2001).

[11] W.A.Bardeen, E.J.Eichten, C.T.Hill, Phys. Rev. D 68, 054024 (2003).

[12] K.D.Chao, Phys. Lett. B 599,43 (2004). 
[13] E.S.Swanson, Phys. Rept. 429, 243 (2006).

[14] P.Colangelo, F.De Fazio, R.Ferrandes, Mod. Phys. Lett. A 19,2083 (2004);P.Colangelo, F.De Fazio, Phys. Lett. B 570,180 (2003), hep-ph/0609072.

[15] E.van Beveren, G. Rupp, Phys. Rev. Lett. 91, 012003 (2003); Mod. Phys. Lett. A 19, 1949 (2004).

[16] D.S. Hwang, D.W. Kim Phys. Lett. B 601, 137 (2004).

[17] F.L. Wang, X.L.Chen, D.H. Lu, S.L. Zhu, W.Z.Deng, hep-ph/0604090 Y.B. Dai, S.L. Zhu, Y.B.Zuo, hep-ph/0610327.

[18] Yu.A. Simonov, J.A.Tjon, Phys. Rev. D 70, 114013 (2004).

[19] J.Vijande, A.Valcarse, F.Fernandez, arXiv:0711.2359.

[20] Yu.A. Simonov, Phys. Rev. D 65, 094018 (2002).

[21] S. Weinberg, Phys. Rev. Lett. 67 , 3473 (1991).

[22] J.L. Goity, W. Roberts, Phys. Rev. D60, 034001 (1999) and references therein; H.Georgi, A.Manohar, Nucl. Phys. B 234,189 (1984).

[23] Yu.A. Simonov, Phys. At. Nucl. 60, 2069 (1997); hep-ph/9704301;

Yu.A. Simonov, J.A. Tjon, Phys. Rev. D 62, 014501 (2000), ibid.D 62, 094511 (2000).

[24] A.Yu. Dubin, A.B. Kaidalov,Yu.A. Simonov, Phys. Atom. Nucl. 56, 1795 (1993); Phys. Lett. B 323, 41 (1994); E.Gubankova, A.Yu. Dubin, Phys. Lett. B 324, 180 (1994).

[25] A.M. Badalian, B.L.G. Bakker, Yu.A. Simonov, Phys. Rev. D 75, 116001, 2007.

[26] Particle Data Group, J. of Phys. G 32, 1 (2006).

[27] V.M.Abazov et al., (D0 Collab.), arXiv:0705.3229.

[28] T. Aaltonen et al., (CDF Collaboration), arXiv: 0710.4199: R.K.Mommsen, hep-ex/0612003

I.V.Gorelov, hep-ex/0610080; P.Catastini hep-ex/0605051.

[29] R.N.Cahn, J.D.Jackson, Pys.Rev. D68, 037502 (2003).

[30] O.Aquines et al (CLEO) Phys. Rev. Lett. 96, 152001 (2006);

G.Bonvicini et al (CLEO) Phys. Rev. Lett. 96, 022002 (2006).

[31] A.M.Badalian, B.L.G.Bakker, Phys. Rev. D 62, 094031 (2000);

A.M.Badalian, B.L.G.Bakker, V.L.Morgunov, Phys. Rev. D 60, 116008 (1999).

[32] Y.Koma, M.Koma, hep-lat/0609076 (2006)

[33] A.M.Badalian, L.P.Kok, M.I.Polikarpov, Yu.A.Simonov, Phys. Rept. 82, 32 (1982).

[34] H.Feshbach, Ann. Phys. (N.Y.) 5, 357 (1958). 
[35] A.F.Falk, T.Mehen, Phys. Rev. D 53, 231 (1996). P.L.Cho, M.B.Wise, Phys. Rev. 49, 6228 (1994).

[36] A. Zghiche ( for BaBar Collaboration), arXiv:0710.0314.

[37] A.M.Badalian, B.L.G.Bakker, hep-ph/0702229, JETP Lett. 86 (2007).

[38] Yu.A.Simonov, Phys. Lett. B 515,137 (2001).

[39] Yu.A.Simonov and M.A.Trusov, hep-ph/0506058, hep-ph/0607075.

\section{APPENDIX A: CONNECTION BETWEEN THE CHIRAL QUARK-PION LA- GRANGIAN AND THE EFFECTIVE CHIRAL LAGRANGIAN}

The interaction of pions with quarks was introduced and developed in [21], [22], see [17] for recent applications. The effective chiral Lagrangian $\Delta L_{e f f}$ contains one new parameter $g_{A}^{q}$,

$$
L_{\pi q}=\frac{g_{A}^{q}}{2 f_{\pi}} \bar{\Psi} \gamma_{\mu} \gamma_{5} \tau \Psi \partial^{\mu} \phi_{\pi}
$$

and has the form of pseudovector coupling, known from phenomenological applications in the pion-nucleon systems. As was argued in [21], $g_{A}^{q}$ at large $N_{c}$ tends to unity.

In Eq.(17) we have used another form of the quark-pion interaction, derived directly from the QCD Lagrangian in [20] and not containing new parameters,

$$
\Delta L_{F C M}^{(1)}=\int \bar{\psi}(x) \sigma|\mathbf{x}| \gamma_{5} \frac{\pi^{a} \lambda^{a}}{f_{\pi}} \psi(x) d^{4} x
$$

In [20] the connection between (A1) and (A2) was established and here we repeat the derivation for the convenience of readers.

Consider application of (A2) to the case of pionic transition between states $\psi_{1}(x)$ and $\psi_{2}(x)$ of the quark in heavy-light meson. Dirac equations for $\psi_{i}(x)$ can be written as

$$
\begin{gathered}
\left(\boldsymbol{\alpha} \mathbf{p}+\beta(m+\sigma|\mathbf{x}|)+V_{\text {coul }}\right) \psi_{1}=\varepsilon_{1} \psi_{1} \\
\bar{\psi}_{2}\left(-\boldsymbol{\alpha} \mathbf{p}+\beta(m+\sigma|\mathbf{x}|)+V_{\text {coul }}\right)=\varepsilon_{2} \psi_{2} .
\end{gathered}
$$

Expressing in (3), (A4) the term $\sigma|\mathbf{x}| \gamma_{5}$ via $\boldsymbol{\alpha} \mathbf{p}, \beta m$ etc. and summing two equations, one gets

$$
\Delta L_{F C M}^{(1)}=\frac{1}{2 f_{\pi}} \bar{\psi}_{2}\left(-2 m \gamma_{5} \hat{\pi}+\beta \gamma_{5}\left(\varepsilon_{2}-\varepsilon_{1}\right) \hat{\pi}+\gamma_{5} \beta \boldsymbol{\alpha} \mathbf{p} \hat{\pi}\right) \psi_{1}
$$


Since $\gamma_{i}=-i \beta \alpha_{i}$, and $\left(\varepsilon_{2}-\varepsilon_{1}\right) \hat{\pi}=i \frac{\partial}{\partial t} \hat{\pi}(t) \hat{\pi}(t) \sim e^{-i\left(\varepsilon_{2}-\varepsilon_{1}\right) t}$, one can rewrite the last two terms in (A5) as $\gamma_{\mu} \gamma_{5} \partial_{\mu} \hat{\pi}$, and finally one arrives at

$$
\Delta L_{F C M}^{(1)}=\frac{1}{2 f_{\pi}} \bar{\psi}_{2}\left(-2 m \gamma_{5} \hat{\pi}+\gamma_{\mu} \gamma_{5} \partial_{\mu} \hat{\pi}\right) \psi_{1} .
$$

Comparing (A1) and (A6), one can see that in the chiral limit, $m_{q} \rightarrow 0$, two expressions coincide. However, for nonzero $m$, e.g. for strange quark having the mass $m_{s} \sim 0.2 \mathrm{GeV}$ at low scale $\sim 1 \mathrm{GeV}$ [37], first term in (A6) is becoming essential. Moreover, our expression (A2) is only the first term in the expansion of the exponent (15) in powers of the pion field, and therefore this general Lagrangian can be used for decay channels with the production of two or several pions.

\section{APPENDIX B: MASSES OF HEAVY-LIGHT MESONS}

To calculate masses and different matrix elements (m.e.) of a HL meson $(q \bar{b}, q \bar{c}$, or $\bar{q} b)$ we use here the relativistic string Hamiltonian $\hat{H}_{\omega}$, derived in [24]. For this Hamiltonian the spin-averaged mass $M_{c o g}(n L)$ is given by simple formula:

$$
M_{c o g}(n L)=M_{0}(n L)+\Delta_{S E}+\Delta_{s t r},
$$

where $M_{0}$ is the eigenvalue (e.v.) of the spin-independent part $H_{0}$ of the Hamiltonian $\hat{H}_{\omega}$, which coincides with well-known spinless Salpeter Hamiltonian (SSH):

$$
\begin{gathered}
H_{0}=\sqrt{\mathbf{p}^{2}+m_{q}^{2}}+\sqrt{\mathbf{p}^{2}+m_{b}^{2}}+V_{0}(r), \\
H_{0} \varphi_{n L}(r)=M_{0} \varphi_{n l}(r) .
\end{gathered}
$$

However, the mass (B1) contains negative (string) correction and therefore in our approach for a given static potential the levels with $L \neq 0$ lie lower than for SSH. Also the mass (B1) does not contain an overall fitting constant but takes into account NP self-energy term $\Delta_{S E}$ for a light quark ( which is calculated explicitly in [38]).

The static potential

$$
V_{0}(r)=\sigma r-\frac{4}{3} \frac{\alpha_{B}(r)}{r}
$$

is taken here from [25] with the vector oupling $\alpha_{B}(r)$ for $n_{f}=3$. The solutions of (B33) define $M_{0}(n L)$ and m.e., in particular,

$$
\omega_{q}(n L)=\left\langle\sqrt{\mathbf{p}^{2}+m_{q}^{2}}\right\rangle_{n L}, \quad \omega_{Q}(n L)=\left\langle\sqrt{\mathbf{p}^{2}+m_{Q}^{2}}\right\rangle_{n L}
$$


TABLE X: The constituent masses $\omega_{q}$ and $\omega_{b}$ (in $\left.\mathrm{MeV}\right)$ for the $B(1 P)$ and $B_{s}(1 P)$ mesons $\left(m_{u(d)}=\right.$ $\left.0, m_{s}=200 \mathrm{MeV}, m_{b}=4780 \mathrm{MeV}\right)$

\begin{tabular}{|c|c|c|}
\hline & $B(1 P)$ meson & $B_{s}(1 P)$ meson \\
\hline$\omega_{q}(1 P)$ & 680 & 730 \\
\hline$\omega_{b}(1 P)$ & 4836 & 4840 \\
\hline$\omega_{\text {red }}$ & 598 & \\
& & \\
\hline
\end{tabular}

which apear to be the dynamical (constituent) mass of a light quark $\omega_{q}$ and $\omega_{Q}$ for a heavy quark. Their values for the $B$ and $B_{s}$ mesons are given in Table $\mathrm{X}$ together with the reduced mass: $\omega_{\text {red }}=\frac{\omega_{q} \omega_{b}}{\omega_{q}+\omega_{b}}$.

As seen from Table $\mathrm{X}$ the kinetic energy of a light (strange) quark $\omega_{q}(1 P)$ are not small and this fact is important for the fine structure analysis.

In the mass formula (B1) the correction $\Delta_{S E}$ comes from NP self-energy contribution (which is equal zero for the $b$ quark), taken here in the simplest form when self-energy contribution of the $c$ quark $(\lesssim-15 \mathrm{MeV})$ can be neglected,because it is small as compared to the pole $c$-quark mass known at present with the accuracy $\pm 100 \mathrm{MeV}$ [26]). For a light quark $\Delta_{S E}$ has been defined in [38]:

$$
\Delta_{S E}(n L)=\delta-\frac{1.5 \sigma \eta_{q}}{\pi \omega_{q}}
$$

in which small correction, $\sim 3 \div 6 \mathrm{MeV}$ (defined in [25]) is neglected. The factor $\eta_{u(d)}=1.0$ for a light quark and $\eta_{s}=0.65$ for the $s$ quark with $m_{s}=220 \mathrm{MeV}$.

The string correction $\Delta_{s t r}$ for the $1 P$-wave $B\left(B_{s}\right)$ mesons is equal $\Delta_{s t r} \approx-27(-21)$ 
TABLE XI: The masses $M_{0}, M_{\operatorname{cog}}(1 P)$ and $\Delta_{S E}(1 P), \Delta_{s t r}(1 P)$ (in $\mathrm{GeV}$ ) for the $B, B_{s}$ mesons $\left(m_{s}=220 \mathrm{MeV}, m_{u(d)}=0, m_{b}=4780 \mathrm{MeV}\right)$

\begin{tabular}{|c|c|c|}
\hline & $B(1 P)$ & $B_{s}(1 P)$ \\
\hline$M_{0}(1 P)$ & 5885 & 5925 \\
$\Delta_{S E}$ & -126 & -70 \\
\hline$\Delta_{\text {str }}$ & -27 & -20 \\
\hline$M_{\text {cog }}(1 P)$ & 5.732 & 5.835 \\
\hline
\end{tabular}

$\mathrm{MeV}$ [25]. This negative contribution to $M_{\text {cog }}$ improves an agreement with the experimental masses of $B\left(2^{+}\right)$and $B\left(1^{+}\right)$mesons [28]. In Table XI the eigenvalues $M_{0}(1 P)$ and $M_{\operatorname{cog}}(1 P)$ together with $\Delta_{S E}(1 P)$ and $\Delta_{s t r}(1 P)$ are given.

The difference between the e.v. $M_{0}(B)$ and $M_{0}\left(B_{s}\right)$ is only $\sim 50 \mathrm{MeV}$ for $m_{s}=200 \mathrm{MeV}$, so that additional $50 \mathrm{MeV}$ difference in $M_{\text {cog }}(1 P)$ for the $B$ and $B_{s}$ comes from the self-energy terms.

\section{APPENDIX C: FINE STRUCTURE SPLITTINGS}

To define FS splittings of a HL meson we follow here the approach, where the LS basis is used and the analysis of FS can be done in general terms [29]. If one introduces tensor splittings $t(n P)$ and spin-orbit splitting $a(n P)$ then the masses of the $2^{+}$state $\left(j=\frac{3}{2}\right)$ and 
$0^{+}$state $\left(j=\frac{1}{2}\right)$ can be written as

$$
\begin{gathered}
M\left(2^{+}\right)=M_{c o g}+a-0.1 t, \\
M\left(0^{+}\right)=M_{c o g}-2 a-t,
\end{gathered}
$$

while the $1^{+}$states, ${ }^{3} P_{1}$ and ${ }^{1} P_{1}$, are mixed.

The mixing matrix can be expressed through the splittings $a$ and $t$ :

$$
\hat{O}_{\text {mix }}=\left(\begin{array}{ll}
a-\frac{7}{6} t, & -\frac{\sqrt{2}}{6} t \\
-\frac{\sqrt{2}}{6} t, & -2 a+\frac{5}{3} t
\end{array}\right) .
$$

Then the eigenvalues and eigenvectors of this matrix define "higher" and "lower" masses $M_{H}$ and $M_{L}$ with $J^{P}=1^{+}$and the decomposition of their w.f.. The mass splittings are

$$
\begin{aligned}
& M_{H}=M_{c o g}-\frac{1}{4}(2 a-t)+\frac{1}{4} \sqrt{(2 a-t)^{2}+32(a-t)^{2}} ; \\
& M_{L}=M_{c o g}-\frac{1}{4}(2 a-t)-\frac{1}{4} \sqrt{(2 a-t)^{2}+32(a-t)^{2}} .
\end{aligned}
$$

Each of these levels is a decomposition of the ${ }^{3} P_{1}$ and ${ }^{1} P_{1}$ states. From (C3) it is evident that the weights in those decompositions depend only on the ratio

$$
R=\frac{a}{t}
$$

Just the value of this ratio defines the order of levels inside the $n P$ multiplet and for given $a / t$ the mixing angle $\theta$ in (9) can be easily calculated. With the use of the relation (10) the connection between the mixing angle $\phi$ in the basis $\left|j=\frac{1}{2}\right\rangle, \quad\left|j=\frac{3}{2}\right\rangle$ (see the definition in (6), (7)) and the mixing angle $\theta$ in the $\mathbf{L S}$ scheme, for which

$$
\left|1_{H}^{+}\right\rangle=\cos \theta\left|{ }^{3} P_{1}\right\rangle-\sin \theta\left|{ }^{1} P_{1}\right\rangle
$$

have been established: $\phi=-\theta+35.264^{\circ}$.

The value $a / t=1.0 \pm 0.05$ provides large mass shifts of the $D_{s}\left(1_{H}^{+}\right)$and $B_{s}\left(1_{H}^{+}\right)$levels and at the same time keeps the position of the $D_{s}\left(1_{L}^{+}\right)$and $B_{s}\left(1_{L}^{+}\right)$unchanged (with accuracy $2 \mathrm{MeV}$ ). Notice, that for all multiplets with $a / t=0.95$ the mass difference between two narrow levels is

$$
\begin{gathered}
M\left(2^{+}\right)-M\left(1_{L}^{+}\right)=1.31 t \text { for } a / t=0.95, \\
M\left(2^{+}\right)-M\left(1_{L}^{+}\right)=1.40 t \text { for } a / t=1.0
\end{gathered}
$$


so that this relation can be used to define the parameter $t$ from experiment.

To interpret this splitting $t$ one can use well-known perturbative expression, taking onegluon-exchange interaction and neglecting higher in $\alpha_{s}$ corrections, which however may be important [31]:

$$
t(n P)=\frac{4}{3} \frac{\alpha_{F S}\left\langle r^{-3}\right\rangle_{n P}}{\omega_{q} \omega_{Q}} .
$$

Then for $\alpha_{F S}=0.39$ one obtains the values: $t=11.7 \mathrm{MeV}$ for the $B_{s}$ mesons $\left(\left\langle r^{-3}\right\rangle_{1 P}=\right.$ $\left.0.080 \mathrm{GeV}^{3}\right)$ and $t=12.1$ for the $B(1 P)$ mesons $\left(\left\langle r^{-3}\right\rangle_{1 P}=0.0765\right.$, which are close to those, used in our analysis. For the $D(1 P)$ and $D_{s}(1 P)$ meson the same $\alpha_{F S}=0.39$ gives $t_{D}=t_{D_{s}}=29 \mathrm{MeV}\left(\left\langle r^{-3}\right\rangle_{D}=0.052\right.$ and $\left.\left\langle r^{-3}\right\rangle_{D_{s}}=0.055 \mathrm{GeV}^{3}\right)$.

However, in OGE approximation the situation with SO splitting is not so simple.This splitting can be presented in the convenient form from [29] (here we also keep the term proportional $m_{Q}^{-2}$ :

$$
a(n P)=\left(\frac{1}{4 \omega_{q}^{2}}+\frac{1}{4 \omega_{Q}^{2}}\right) A(n P)+t(n P),
$$

where the factor

$$
A(n P)=\frac{4}{3} \alpha_{F S}\left\langle r^{-3}\right\rangle_{n P}-\sigma\left\langle r^{-1}\right\rangle_{n P}
$$

To satisfy the condition $a=0.95 t$ the term $A(1 P)$ has to be small as compared with $t$. However, in OGE approximation and for linear confinement this term in (C10) appears to be negative and not small for $\alpha_{F S} \sim 0.39$. The reason for that needs a special investigation and can mean that either higher radiative corrections are important [31], or a suppression of confining potential in spin-orbit term ,observed on the lattice [32], is essential.

Notice that the Coulomb-type order of levels, i.e. $M\left(0^{+}\right)<M\left(1_{L}^{+}\right)<M\left(1_{H}^{+}\right)<M\left(2^{+}\right)$, takes place only for not small ratio $\frac{a}{t} \geq 0.606$. In our case with $\frac{a}{t}=0.95$ this condition is satisfied and the level $1_{H}^{+}$lies below the $2^{+}$level,

$$
M_{H}\left(1^{+}\right)<M\left(2^{+}\right)
$$

Just this order of levels is observed in the $D(1 P)$ multiplet where the central value of the wide $1^{+^{\prime}}$ level is smaller than the mass of $2^{+}$state [26]. The FS splittings of the $P$-wave HL mesons with $a / t=0.95$ are given in Tables II, III, V] 